\title{
On Mutually Orthogonal Graph-Path Squares
}

\author{
Ramadan El-Shanawany \\ Department of Physics and Engineering Mathematics, Faculty of Electronic Engineering, Menoufia University, \\ Menouf, Egypt \\ Email: Ramadan_elshanawany380@yahoo.com
}

Received 14 January 2015; accepted 14 December 2015; published 17 December 2015

Copyright (C) 2016 by author and Scientific Research Publishing Inc.

This work is licensed under the Creative Commons Attribution International License (CC BY).

http://creativecommons.org/licenses/by/4.0/

(c) $\underset{\mathrm{EY}}{\mathrm{EY}}$ Open Access

\section{Abstract}

A decomposition $\mathcal{G}=\left\{G_{0}, G_{1}, \cdots, G_{s-1}\right\}$ of a graph $H$ is a partition of the edge set of $H$ into edgedisjoint subgraphs $G_{0}, G_{1}, \cdots, G_{s-1}$. If $G_{i} \cong G$ for all $i \in\{0,1, \cdots, s-1\}$, then $\mathcal{G}$ is a decomposition of $H$ by $G$. Two decompositions $\mathcal{G}=\left\{G_{0}, G_{1}, \cdots, G_{n-1}\right\}$ and $\mathcal{F}=\left\{F_{0}, F_{1}, \cdots, F_{n-1}\right\}$ of the complete bipartite graph $K_{n, n}$ are orthogonal if, $\left|E\left(G_{i}\right) \cap\left(F_{j}\right)\right|=1$ for all $i, j \in\{0,1, \cdots, n-1\}$. A set of decompositions $\left\{\mathcal{G}_{0}, \mathcal{G}_{1}, \cdots, \mathcal{G}_{k-1}\right\}$ of $K_{n, n}$ is a set of $k$ mutually orthogonal graph squares (MOGS) if $\mathcal{G}_{i}$ and $\mathcal{G}_{j}$ are orthogonal for all $i, j \in\{0,1, \cdots, k-1\}$ and $i \neq j$. For any bipartite graph $G$ with $n$ edges, $N(n, G)$ denotes the maximum number $k$ in a largest possible set $\left\{\mathcal{G}_{0}, \mathcal{G}_{1}, \cdots, \mathcal{G}_{k-1}\right\}$ of MOGS of $K_{n, n}$ by $G$. Our objective in this paper is to compute $N(n, G)$ where $G=\mathbb{P}_{d+1}(F)$ is a path of length $d$ with $d+1$ vertices (i.e. Every edge of this path is one-to-one corresponding to an isomorphic to a certain graph $F$ ).

\section{Keywords}

Orthogonal Graph Squares, Orthogonal Double Cover

\section{Introduction}

In this paper we make use of the usual notation: $K_{m, n}$ for the complete bipartite graph with partition sets of sizes $m$ and $n, P_{n+1}$ for the path on $n+1$ vertices, $D \bigcup F$ for the disjoint union of $D$ and $F, D \bigcup^{L_{v}} F$ for the union of $D$ and $F$ with $L_{v}$ (set of vertices) that belong to each other (i.e. union of $D$ and $F$ with common vertices of the set $L_{v}$ belong to $F$ and $D$ ), $K_{n}$ for the complete graph on $n$ vertices, $K_{1}$ for an isolated vertex. The other terminologies not defined here can be found in [1].

A decomposition $\mathcal{G}=\left\{G_{0}, G_{1}, \cdots, G_{s-1}\right\}$ of a graph $H$ is a partition of the edge set of $H$ into edge-disjoint sub- 
graphs $G_{0}, G_{1}, \cdots, G_{s-1}$. If $G_{i} \cong G$ for all $i \in\{0,1, \cdots, s-1\}$, then $\mathcal{G}$ is a decomposition of $H$ by $G$. Two decompositions $\mathcal{G}=\left\{G_{0}, G_{1}, \cdots, G_{n-1}\right\}$ and $\mathcal{F}=\left\{F_{0}, F_{1}, \cdots, F_{n-1}\right\}$ of the complete bipartite graph $K_{n, n}$ are orthogonal if, $\left|E\left(G_{i}\right) \cap\left(F_{j}\right)\right|=1$ for all $i, j \in\{0,1, \cdots, n-1\}$. Orthogonality requires that $\left|E\left(G_{i}\right)\right|=n=\left|E\left(F_{i}\right)\right|$ for all $i \in\{0,1, \cdots, n-1\}$. A set of decompositions $\left\{\mathcal{G}_{0}, \mathcal{G}_{1}, \cdots, \mathcal{G}_{k-1}\right\}$ of $K_{n, n}$ is a set of $k$ mutually orthogonal graph squares (MOGS) if $\mathcal{G}_{i}$ and $\mathcal{G}_{j}$ are orthogonal for all $i, j \in\{0,1, \cdots, k-1\}$ and $i \neq j$. We use the notation $N(n, G)$ for the maximum number $k$ in a largest possible set $\left\{\mathcal{G}_{0}, \mathcal{G}_{1}, \cdots, \mathcal{G}_{k-1}\right\}$ of MOGS of $K_{n, n}$ by $G$, where $G$ is a bipartite graph with $n$ edges.

If two decompositions $\mathcal{G}$ and $\mathcal{F}$ of $K_{n, n}$ by $G$ are orthogonal, then $\mathcal{G} \cup \mathcal{F}$ is an orthogonal double cover of $K_{n, n}$ by $G$. Orthogonal decompositions of graphs and orthogonal double covers (ODC) of graphs have been studied by several authors; see the survey articles [2] [3].

It is well-known that orthogonal Latin squares exist for every $n \notin\{2,6\}$. A family of $k$-orthogonal Latin squares of order $n$ is a set of $k$ Latin squares any two of which are orthogonal. It is customary to denote $N(n)=\max \{k: \exists k M O L S\}$ be the maximal number of squares in the largest possible set of mutually orthogonal Latin squares MOLS of side $n$. A decomposition of $K_{n, n}$ by $n K_{2}$ is equivalent to a Latin square of side $n$; two decompositions $\mathcal{G}$ and $\mathcal{F}$ of $K_{n, n}$ by $n K_{2}$ are orthogonal if and only if the corresponding Latin squares of side $n$ are orthogonal; and thus $N\left(n, n K_{2}\right)=N(n)$. The computation of $N(n)$ is one of the most difficult problems in combinatorial designs; see the survey articles by Abel et al. [4] and Colbourn and Dinitz in [5]. Since $N(n, G)$ is a natural extension of $N(n)$, the study of $N(n, G)$ for general graphs is interesting. El-Shanawany [6] establishes the following: i) $N\left(n, K_{1, n}\right)=2$; ii) $N\left(2, P^{3}\right)=2, N\left(3, P^{4}\right)=3, N\left(5, P_{6}\right)=5$ and $N\left(7, P_{8}\right)=7$; iii) let $p>2$ be a prime number, then $N\left(p, K_{2}+((p-1) / 2) P_{3}\right)=p$; iv) let $p$ be a prime number, then $N\left(p,(p-2) K_{2}+P_{3}\right) \geq p-1$. Based on ii), El-Shanawany [6] proposed:

Conjecturer 1. Let $p$ be a prime number. Then $N\left(p, P_{p+1}\right)=p$.

Sampathkumar et al. [7] have proved El-Shanawany conjectured. In the following section, we present another technique to prove this conjecture as in Theorem 8.

The two sets $\left\{0_{0}, 1_{0}, \cdots,(n-1)_{0}\right\}$ and $\left\{0_{1}, 1_{1}, \cdots,(n-1)_{1}\right\}$ denote the vertices of the partite sets of $K_{n, n}$. The length of the edge $x_{0} y_{1}$ of $K_{n, n}$ is defined to be the difference $y-x$, where $x, y \in \mathbb{Z}_{n}$. Note that sums and differences are carried over in $\mathbb{Z}_{n}$ (that is, sums and differences are carried modulo $n$ ). Let $G$ be a subgraph of $K_{n, n}$ without isolated vertices and let $a \in\{0,1, \cdots, n-1\}$. The $a$-translate of $G$, denoted by $G+a$, is the edgeinduced subgraph of $K_{n, n}$ induced by $\left\{(x+a)_{0}(y+a)_{1}: x_{0} y_{1} \in E(G)\right\}$. A subgraph $G$ of $K_{n, n}$ is half-starter if $|E(G)|=n$ and the lengths of all edges in $G$ are mutually different.

Lemma 2 (see [8]). If $G$ is a half-starter, then the union of all translates of $G$ forms an edge decomposition of $K_{n, n}$ (i.e. $\left.E\left(K_{n, n}\right)=\bigcup_{a \in \mathbb{Z}_{n}} E(G+a)\right)$.

In what follows, we denote a half-starter $G$ by the vector $v(G)=\left(v_{0}, v_{1}, \cdots, v_{n-1}\right) \in \mathbb{Z}_{n}^{n}=\underbrace{\mathbb{Z}_{n} \times \mathbb{Z}_{n} \times \cdots \times \mathbb{Z}_{n}}_{n \text { times }}$, where $v_{0}, v_{1}, \cdots, v_{n-1} \in \mathbb{Z}_{n}$ and $v_{i}$ can be obtained from the unique edge $\left(v_{i}\right)_{0}\left(v_{i}+i\right)_{1}$ of length $i$ in $G$.

Theorem 3 (see [8]). Two half-starters $v(G)=\left(v_{0}, v_{1}, \cdots, v_{n-1}\right) \in \mathbb{Z}_{n}^{n}$ and $v(F)=\left(u_{0}, u_{1}, \cdots, u_{n-1}\right) \in \mathbb{Z}_{n}^{n}$ are orthogonal if $\left\{v_{i}-u_{i}: i \in \mathbb{Z}_{n}\right\}=\mathbb{Z}_{n}$.

If two half-starters $v(G)$ and $v(F)$ are orthogonal, then the set of translates of $G$ and the set of translates of $F$ are orthogonal.

A set of decompositions $\left\{\mathcal{G}_{i}=\bigcup_{a \in \mathbb{Z}_{n}} E\left(G_{i}+a\right)=E\left(K_{n, n}\right): 0 \leq i \leq k-1\right\}$ of $\left.K_{n, n}\right\}$ is a set of $k$ mutually orthogonal graph squares (MOGS) if $\mathcal{G}_{i}$ and $\mathcal{G}_{j}$ are orthogonal for all $i, j \in\{0,1, \cdots, k-1\}$ and $i \neq j$.

Note that

$$
\bigcup_{i=0}^{k-1} \mathcal{G}_{i}=\bigcup_{i=0}^{k-1}\left(\bigcup_{a \in \mathbb{Z}_{n}} E\left(G_{i}+a\right)\right)=k E\left(K_{n, n}\right) .
$$

In the following, we define a $G$-square over additive group $\mathbb{Z}_{n}$.

Definition 4 (see [6]). Let $G$ be a subgraph of $K_{n, n}$ A square matrix $\mathcal{L}$ of order $n$ is called an $G$-square if every element in $\mathbb{Z}_{n}$ occur exactly $n$ times, and the graphs $G_{i}$, $i \in \mathbb{Z}_{n}$ with 
$E\left(G_{i}\right)=\left\{(x, y): \mathcal{L}(x, y)=i, x, y \in \mathbb{Z}_{n}\right\}$ are isomorphic to graph $G$.

We have already from Lemma 2 and Definition 4 that every half starter vector $v(G)$ and its translates are equivalent to $G$-square. For more illustration, the first matrix $\mathcal{L}_{0}$ in equation (1) is equivalent to the first row in Figure 3, which represented by the half starter vector $v\left(G_{00}\right)=(0,0,2)$ and its translates.

Definition 5. Two squares matrices $\mathcal{L}_{0}$ and $\mathcal{L}_{1}$ of order $n$ are said to be orthogonal if for any ordered pair $(a, b)$, there is exactly one position $(x, y)$ for $\mathcal{L}_{0}(x, y)=a$ and $\mathcal{L}_{1}(x, y)=b$.

Now, we shall derive a class of mutually orthogonal subgraphs of $K_{n, n}$ by a given graph $G$ as follow.

Definition 6. A set of matrices $\left\{\mathcal{L}_{i}: 0 \leq i \leq k-1\right\}$ of $K_{n, n}$ is called a set of $k$ mutually orthogonal graph squares (MOGS) if $\mathcal{L}_{i}$ and $\mathcal{L}_{j}$ are orthogonal for all $i, j \in\{0,1, \cdots, k-1\}$ and $i \neq j$.

Definition 7 (see [9]). Let $F$ be a certain graph, the graph $F$-path denoted by $\mathbb{P}_{d+1}(F)$, is a path of a set of vertices $\mathbb{V}=\left\{\mathbb{V}_{i}: 0 \leq i \leq d\right\}$ and a set of edges $\mathbb{E}=\left\{\mathbb{E}_{i}: 0 \leq i \leq d-1\right\}$ if and only if there exists the following two bijective mappings:

1) $\Psi: \mathbb{E} \rightarrow \mathcal{F}$ defined by $\Psi\left(\mathbb{E}_{i}\right)=F_{i}$, where $\mathcal{F}=\left\{F_{0}, F_{1}, \cdots, F_{d-1}\right\}$ is a collection of $d$ graphs, each one is isomorphic to the graph $F$.

2) $\phi: \mathbb{V} \rightarrow \mathcal{Y}$ defined by $\phi\left(\mathbb{V}_{i}\right)=X_{i}$, where $\mathcal{Y}=\left\{X_{i}: 0 \leq i \leq d ; \bigcap_{i} X_{i}=\varnothing\right\}$ is a class of disjoint sets of vertices (i.e., $\mathcal{Y}$ decomposed into $d+1$ disjoint sets such that no two vertices within the same set are adjacent).

As a special case if the given graph $F$ is isomorphic to $K_{1,1}$ then $\mathbb{P}_{d+1}\left(K_{1,1}\right)$, is the natural path $P_{d+1}$ that is, $\mathbb{P}_{d+1}\left(K_{1,1}\right)=P_{d+1}$.

For more illustration, see Figure 1, Figure 2.

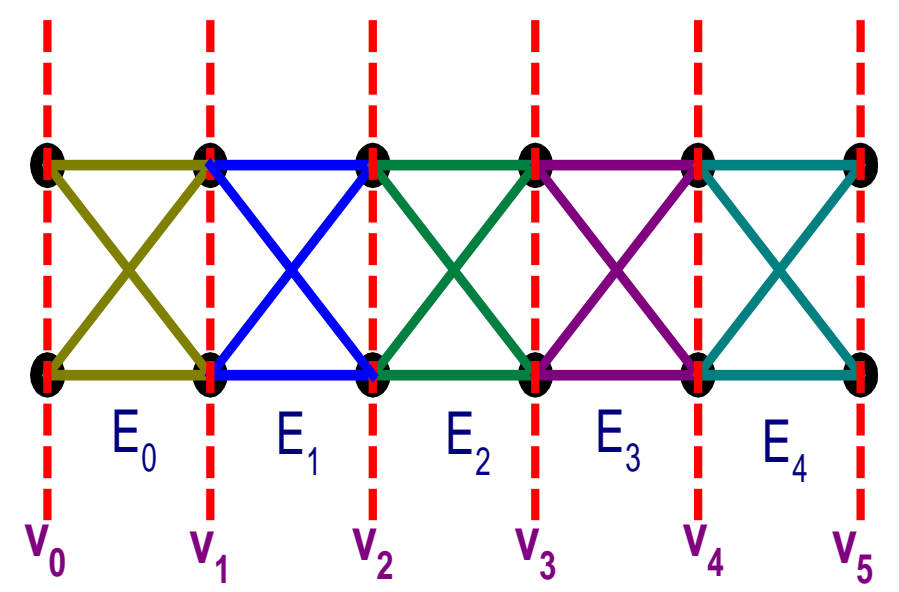

Figure 1. $\mathbb{P}_{6}\left(K_{2,2}\right)$, the path of 6 sets of vertices (every sethas only 2 disjoint vertices) and 5 edges of $K_{2,2}$.

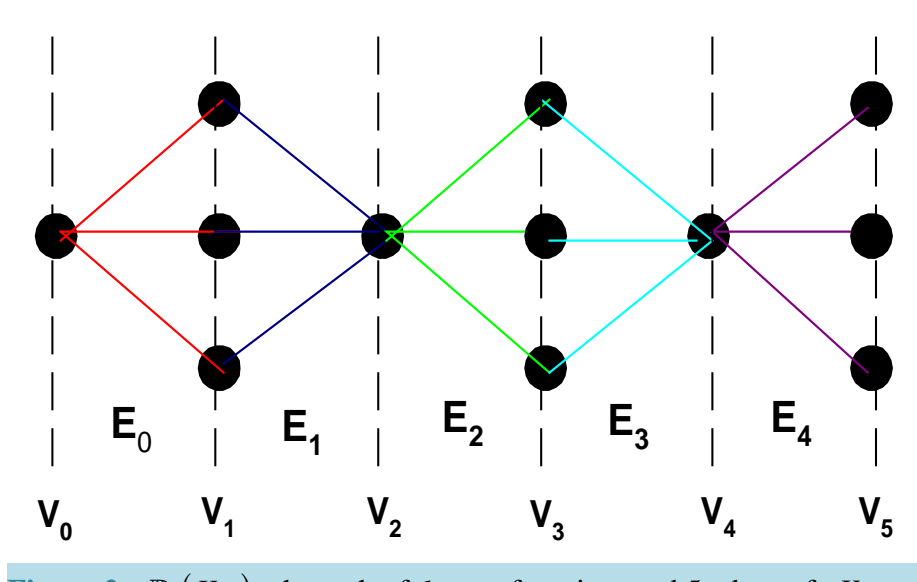

Figure 2. $\mathbb{P}_{6}\left(K_{1,3}\right)$, the path of 6 sets of vertices and 5 edges of $K_{1,3}$. 
Consider $s \geq 0$ paths of length $k \geq 1$, all attached to the same vertex (root vertex). This tree will be called $T(s, k)$. Clearly, $T(s, 1)$ is the star with s edges and $T(1, k)$ is the path with $k$ edges. Define $L_{s}$ as a set of all leaves of $T(s, k)$ i.e. $L_{s}=\{v: v$ is a leaf in $T(s, k)\}$ and $\left|L_{-}\{s\}\right|=s$.

In the following section, we will compute $N(n, G)$ where $G=\mathbb{P}_{d+1}(F)$ such that $F=K_{1,1}$ as in theorem 8 and $F=T(3,3) \bigcup^{L_{3}} K_{1}$ as in theorem 11 .

\section{Mutually Orthogonal Graph-Path Squares}

The following result was shown in [7]. Here we present another technique for the proof.

Theorem 8. Let $q$ be a prime number. Then $N\left(q, \mathbb{P}_{q+1}\left(K_{1,1}\right)\right)=q$.

Proof. Let $G_{i j}$ be a subgraph of $K_{q, q}$ with $q$ edges; for fixed $j \in \mathbb{Z}_{q}$ and $0 \leq i \leq q-1$, define the $q$ halfstarter vectors as follows, $v\left(G_{i j}\right)=\left(j, i+j-1,2 i+j-2^{2}, \cdots,(-2)(i+2)+j,(-1)(i+1)+j\right)$; our task is to prove the orthogonality of those $q$ half-stater vectors in mutually. Let us define the half starter vector $v\left(G_{i j}\right)$ as $v_{k}\left(G_{i j}\right)=k(i-k)+j$ for all $k \in \mathbb{Z}_{q}$. Then for all two different elements $k, l \in \mathbb{Z}_{q}$, we have $\left\{v_{k}\left(G_{i j}\right)-v_{l}\left(G_{i j}\right)=k(i-k)-l(i-l)=(k-l)(i-(k+l))\right\}$, then $v_{k}, v_{l}$ are mutually orthogonal half-starter vectors of graphs $G_{k j}$ and $G_{l j}$ of $K_{q, q}$ respectively iff $(i-(k+l), q)=1$. It remains to prove the isomorphism of $v_{k}\left(G_{i j}\right)$ half starter graphs $G_{i j}$ of $K_{q, q}$ for all $k \in \mathbb{Z}_{q}$. Let

$v_{k}\left(G_{i j}\right)=k(i-k)+j=v_{k}\left(G_{i s}\right)=k(i-k)+s$, and therefore $j=s$. Furthermore, if

$v_{k}\left(G_{i j}\right)=k(i-k)+j=v_{l}\left(G_{i j}\right)=l(i-l)+j$, then $(k-l)(i-(k+l))=0$, since $i-(k+l) \neq 0$ (orthogonality of $v_{k}$ and $\left.v_{l}\right)$, and therefore $k=l$. Moreover, for any $i, j \in \mathbb{Z}_{q}$ the $i j^{\text {th }}$ graph isomorphic to $G_{i j}$ has the edges: $E\left(G_{i j}\right)=\left\{\left(-i^{2}+j\right)_{0}(i(1-i)+j)_{1}\right\}$.

An immediate consequence of the Theorem 8 and Conjecture 1 is the following result.

Example 9. The three mutually orthogonal decompositions (MOD) of $K_{3,3}$ by $P_{4}$ given in Figure 3 are associated with the three mutually orthogonal $P_{4}$-squares as in Equation (1):

$$
\mathcal{L}_{0}=\left[\begin{array}{lll}
0 & 0 & 1 \\
2 & 1 & 1 \\
2 & 0 & 2
\end{array}\right], \mathcal{L}_{1}=\left[\begin{array}{lll}
0 & 2 & 2 \\
0 & 1 & 0 \\
1 & 1 & 2
\end{array}\right], \quad \mathcal{L}_{2}=\left[\begin{array}{lll}
0 & 1 & 0 \\
1 & 1 & 2 \\
0 & 2 & 2
\end{array}\right] .
$$

Note that, every row in Figure 3 represents edge decompositions of $K_{3,3}$ by $P_{4}$.
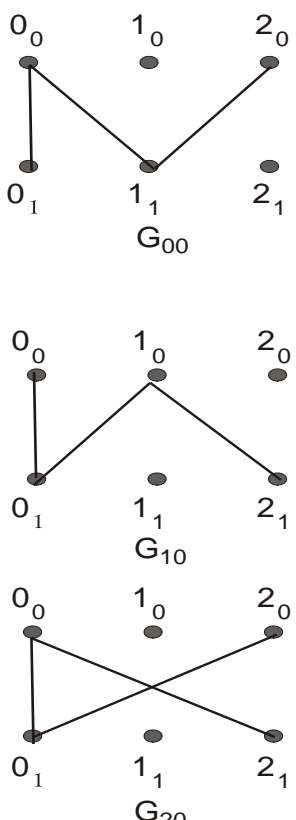
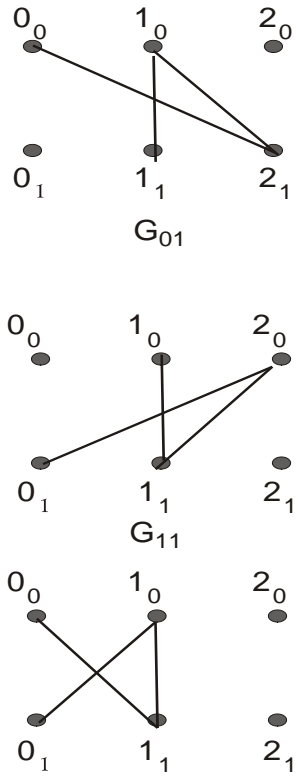

$\mathrm{G}_{21}$
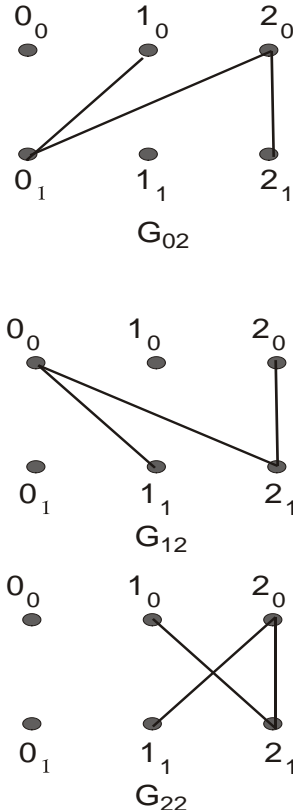

Figure 3. 3MOD of $K_{3,3}$ by $P_{4}$. 


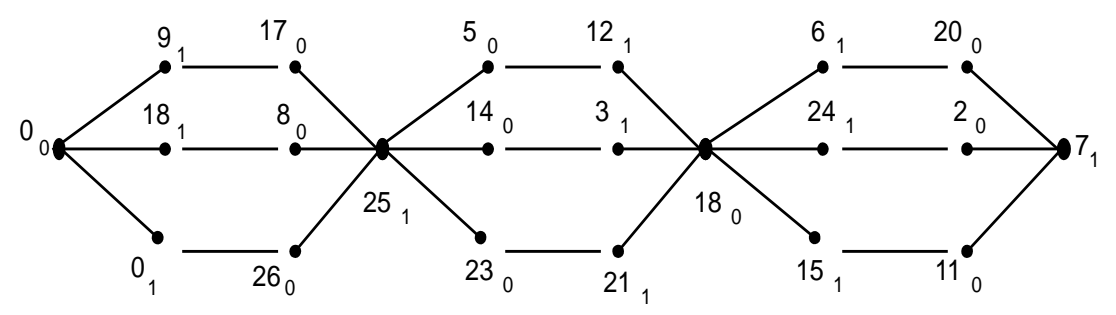

Figure 4. $\mathbb{P}_{4}\left(T(3,3) \bigcup^{L_{3}} K_{1}\right)$, the path of 4 vertices and 3 edges of $T(3,3) \bigcup^{L_{3}} K_{1}$.

The following result is a generalization of the Theorem 8 .

Theorem 10. Let $n$ be a prime power such that $n=q^{x}$ with integer power $x \geq 1$ of a prime number $q$ and $G$ be a subgraph of $K_{n, n}$. Then $N(n, G) \geq q$.

Proof. For fixed $j \in \mathbb{Z}_{n}, 0 \leq i \leq q-1$ and $G_{i j} \cong G$, define the $q$ half-starter vectors as follows,

$$
v\left(G_{i j}\right)=\left(j, i+j-1,2 i+j-2^{2}, \cdots,(-2)(i+2)+j,(-1)(i+1)+j\right) .
$$

Our task is to prove the orthogonality of those $q$ half-starter vectors in mutually. Let us define the half starter vector $v\left(G_{i j}\right)$ as $v_{k}\left(G_{i j}\right)=k(i-k)+j$ for all $k \in \mathbb{Z}_{q}$. Then for all two different elements $k, l \in \mathbb{Z}_{q}$, we have $\left\{v_{k}\left(G_{i j}\right)-v_{l}\left(G_{i j}\right)=k(i-k)-l(i-l)=(k-l)(i-(k+l))\right\}$, and then $v_{k}, \quad v_{l}$ are mutually orthogonal half-stater vectors of graphs $G_{k j}$ and $G_{l j}$ of $K_{n, n}$ respectively iff $(i-(k+l), n)=1$. It remains to prove the isomorphism of $v_{k}\left(G_{i j}\right)$ half starter graphs $G_{i j}$ of $K_{n, n}$ for all $k \in \mathbb{Z}_{q}$. Let

$v_{k}\left(G_{i j}\right)=k(i-k)+j=v_{k}\left(G_{i s}\right)=k(i-k)+s$, and therefore $j=s$. Furthermore, if

$v_{k}\left(G_{i j}\right)=k(i-k)+j=v_{l}\left(G_{i j}\right)=l(i-l)+j$, then $(k-l)(i-(k+l))=0$, since $i-(k+l) \neq 0$ (orthogonality of $v_{k}$ and $v_{l}$ ), and therefore $k=l$. Moreover, for any $0 \leq i \leq q-1, j \in \mathbb{Z}_{n}$ the $i j^{\text {th }}$ graph $G_{i j}$ isomorphic to $G$ has the edges:

$$
E\left(G_{i j}\right)=\left\{\left(n-i^{2}+j\right)_{0}(n-i(i-1)+j)_{1}\right\}
$$

Note that, in the special case $x=1$ the Theorem 10 proved El-Shanawany conjecture; also, in the case $q=2, x \neq 1$, and $G \neq 2 K_{2}$, Theorem 10 constructed an orthogonal double cover of $K_{n, n}$ by $G$.

Furthermore, we can construct the following result using Theorem 10 in case $x>1$ and $q=3$.

Theorem 11. Let $x \geq 2$ be a positive integer such that $n=3^{x}$ and $\mathbb{P}_{3^{x-2}+1}\left(T(3,3) \cup^{L_{3}} K_{1}\right)$ be a subgraph of $K_{3^{x}, 3^{x}}$. Then $N\left(3^{x}, \mathbb{P}_{3^{x-2}+1}\left(T(3,3) \bigcup^{L_{3}} K_{1}\right)\right) \geq 3$.

Proof. The result follows from the vector in Equation (2) and its edges in Equation (3) with $G=\mathbb{P}_{d+1}(F), F=T(3,3) \bigcup^{L_{3}} K_{1}$ such that $|E(G)|=3^{x}=|E(F)| d=9 d$, imply that $d=3^{x-2}$ which define the number of graphs isomorphic to $F$. As a direct application of Theorem 11; see Figure 4.

Conjecture 12. $N\left(q^{x}, \mathbb{P}_{q+1}\left(K_{1,1}\right)\right)=q^{x}$ if $q$ is a prime number with an integer power $x \geq 1$.

Conjecture 13. $N\left(q^{x}, \mathbb{P}_{\frac{q^{x}}{s k}}\left(T(3,3) \cup \cup^{L_{3}} K_{1}\right)\right) \geq q$ if $q$ is a prime number with an integer power $x \geq 1$ and $s, k$ are positive integers.

\section{References}

[1] Balakrishnan, R. and Ranganathan, K. (2012) A Textbook of Graph Theory. Springer, Berlin. http://dx.doi.org/10.1007/978-1-4614-4529-6

[2] Alspach, B., Heinrich, K. and Liu, G. (1992) Orthogonal Factorizations of Graphs. In: Dinitz, J.H. and Stinson, D.R., Eds., Contemporary Design Theory, Chapter 2, Wiley, New York, 13-40.

[3] Gronau, H.-D.O.F., Hartmann, S., Grüttmüller, M., Leck, U. and Leck, V. (2002) On Orthogonal Double Covers of 
Graphs. Designs, Codes and Cryptography, 27, 49-91. http://dx.doi.org/10.1023/A:1016546402248

[4] Colbourn, C.J. and Dinitz, J.H. (eds.) (2007) Handbook of Combinatorial Designs. 2nd Edition, Chapman \& Hall/CRC, London, Boca Raton.

[5] Colbourn, C.J. and Dinitz, J.H. (2001) Mutually Orthogonal Latin Squares: A Brief Survey of Constructions. Journal of Statistical Planning and Inference, 95, 9-48. http://dx.doi.org/10.1016/S0378-3758(00)00276-7

[6] El-Shanawany, R. (2002) Orthogonal Double Covers of Complete Bipartite Graphs. Ph.D. Thesis, Universitat Rostock, Rostock.

[7] Sampathkumar, R. and Srinivasan, S. (2009) Mutually Orthogonal Graph Squares. Journal of Combinatorial Designs, 17, 369-373. http://dx.doi.org/10.1002/jcd.20216

[8] El-Shanawany, R., Gronau, H.-D.O.F. and Grüttmüller, M. (2004) Orthogonal Double Covers of $K_{n, n}$ by Small Graphs. Discrete Applied Mathematics, 138, 47-63. http://dx.doi.org/10.1016/S0166-218X(03)00269-5

[9] El-Shanawany, R., Shabana, H. and ElMesady, A. (2014) On Orthogonal Double Covers of Graphs by Graph-Path and Graph-Cycle. LAP LAMBERT Academic Publishing. https://www.lappublishing.com/ 